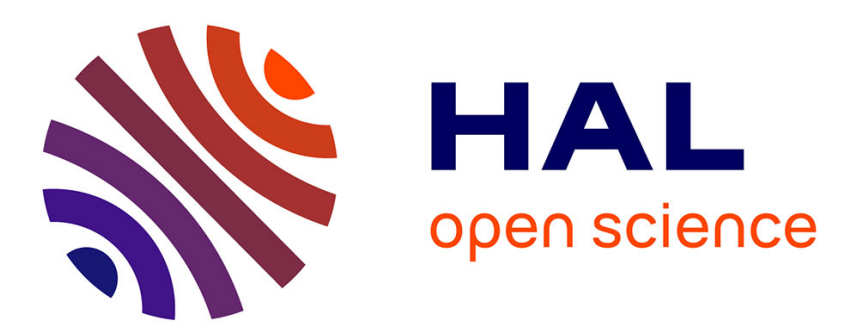

\title{
Etude du Comportement Energétique de l'Explosif à l'Echelle Moléculaire. Approche Théorique et Expérience TRISP
}

D. Delpeyroux, C. Lafon, D. Mathieu, Ph. Simonetti, F. Cansell, D. Fabre, J. Petitet

\section{To cite this version:}

D. Delpeyroux, C. Lafon, D. Mathieu, Ph. Simonetti, F. Cansell, et al.. Etude du Comportement Energétique de l'Explosif à l'Echelle Moléculaire. Approche Théorique et Expérience TRISP. Journal de Physique IV Proceedings, 1995, 05 (C4), pp.C4-521-C4-530. 10.1051/jp4:1995442 . jpa-00253748

\section{HAL Id: jpa-00253748 https://hal.science/jpa-00253748}

Submitted on 1 Jan 1995

HAL is a multi-disciplinary open access archive for the deposit and dissemination of scientific research documents, whether they are published or not. The documents may come from teaching and research institutions in France or abroad, or from public or private research centers.
L'archive ouverte pluridisciplinaire HAL, est destinée au dépôt et à la diffusion de documents scientifiques de niveau recherche, publiés ou non, émanant des établissements d'enseignement et de recherche français ou étrangers, des laboratoires publics ou privés. 


\title{
Etude du Comportement Energétique de l'Explosif à l'Echelle Moléculaire. Approche Théorique et Expérience TRISP
}

\author{
D. Delpeyroux, C. Lafon, D. Mathieu, Ph. Simonetti, F. Cansell*, D. Fabre* and J.P. Petitet* \\ Commissariat à l'Energie Atomique, Centre d'Etudes du Ripault, BP. 16, 37260 Monts, France \\ * LIMHP-CNRS Institut Galilée, 93430 Villetaneuse, France
}

\begin{abstract}
:
The study of energetic behaviour of explosive in relation with its molecular structure needs the analysis of decomposition mechanisms through Quantum Chemistry calculations. We present methodology and first results. The TRISP technique is developed to detect the intermediate chemical species and the detonation products. And the modelling of IR spectra (theoretical calculations and consideration of $(P, T)$ effects on the vibration bands) is a tool to interpret the experimental results.
\end{abstract}

\section{1 - Introduction}

L'objectif de la recherche de nouvelles molécules explosives est l'obtention d'un compromis satisfaisant, pour l'utilisation recherchée, entre les performances énergétiques et les contraintes de sécurité (sensibilité au choc, stabilité thermique). Les performances dépendent en particulier du travail fourni par les gaz de détonation pendant leur détente.

Ainsi, pour chaque molécule appartenant à une famille donnée, la connaissance des réactions chimiques qui se produisent pendant la décomposition et la caractérisation des espèces formées permettent une meilleure compréhension du comportement énergétique du matériau en relation avec sa structure moléculaire et une simulation plus exacte du comportement macroscopique.

L'étude des mécanismes réactionnels mis en jeu pendant la décomposition de l'explosif est réalisée par l'analyse des Surfaces d'Energie Potentielle des réactions chimiques, obtenues par les méthodes de la Chimie Quantique. Nous recherchons une validation expérimentale des résultats théoriques par la mise en évidence des espèces 
formées dans le milieu en détonation à l'aide d'une spectrométrie Infrarouge ultra rapide TRISP [1]. Afin d'aider à l'interprétation de ces spectres Infrarouge expérimentaux, nous utilisons les techniques de calcul de la Chimie Quantique pour leur simulation numérique, et nous exploitons les résultats d'expériences conduites en cellule à enclume de diamant pour évaluer l'influence des effets de forte pression et de température élevée du milieu en détonation sur les bandes de vibration.

\section{2 - Approche théorique}

\section{1 - Modélisation des mécanismes réactionnels de décomposition de l'explosif}

\subsection{1 - Méthodologie}

Nous nous intéressons tout d'abord à un explosif de la famille des Nitramines, l'Hexogène, sur lequel nous disposons d'un grand nombre de résultats d'études concernant sa décomposition. En nous appuyant sur les données recueillies par une recherche bibliographique, nous établissons le diagramme de décomposition de la molécule avec les différentes voies possibles de cassures de liaison et les espèces chimiques résultantes. Nous voyons sur ce diagramme, représenté sur la figure $\mathrm{n}^{\circ} 1$, les trois voies essentielles de décomposition du RDX :

- la rupture de liaison simple $\mathrm{N}-\mathrm{N}$,

- la cassure concertée des liaisons $\mathbf{C}-\mathbf{N}$ du cycle,

- le réarrangement intramoléculaire avec élimination de HONO.

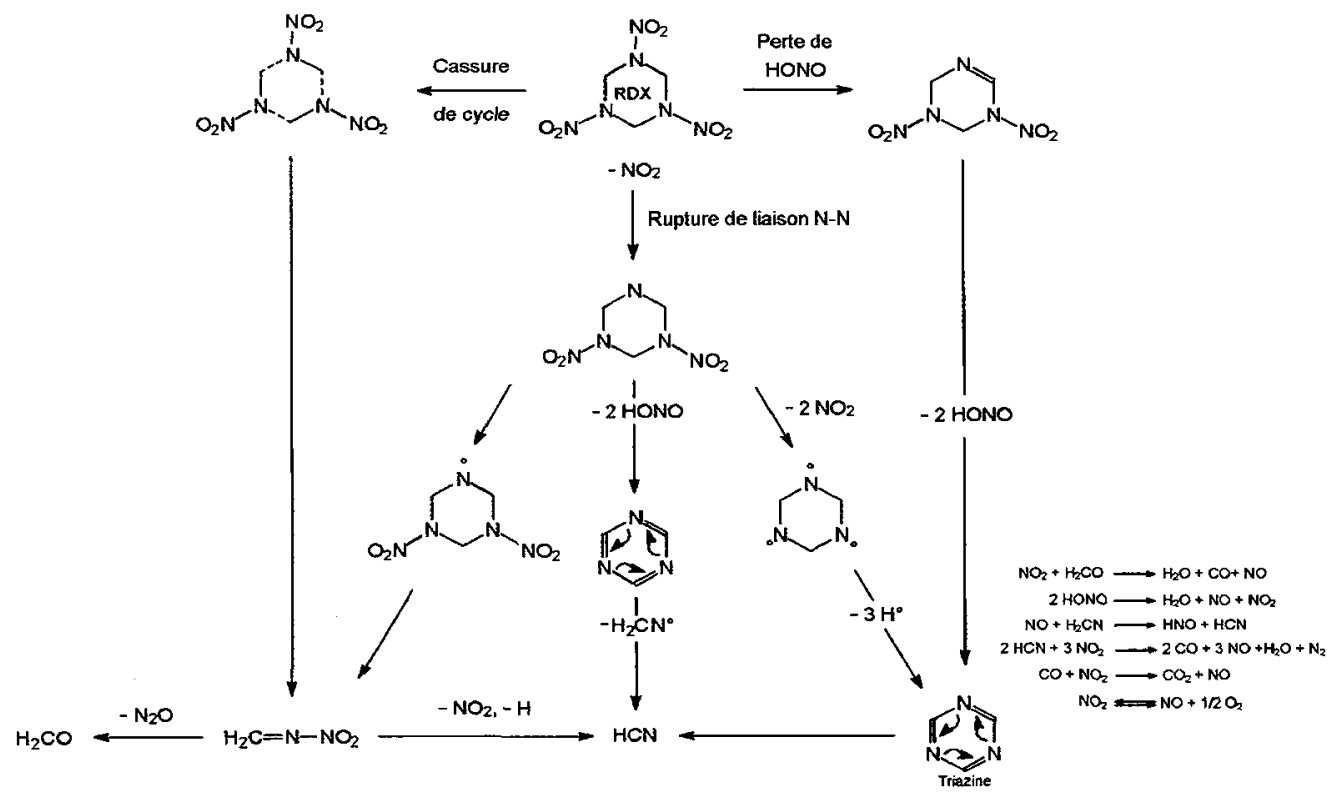

Figure 1 : Schéma de décomposition du RDX 
Ces voies conduisent aux espèces chimiques principales suivantes :

$\mathrm{H}_{2} \mathrm{C}=\mathrm{N}-\mathrm{NO}_{2}$, triazine, $\mathrm{H}_{2} \mathrm{CO}, \mathrm{H}_{2} \mathrm{O}, \mathrm{HCN}, \mathrm{NO}_{2}, \mathrm{HONO}, \mathrm{CO}_{2}, \mathrm{CO}, \mathrm{N}_{2} \mathrm{O}$

Notre objectif est de déterminer l'existence de voies préférentielles parmi celles existantes et de proposer les espèces prépondérantes.

Pour cela, nous localisons sur le graphe de la fonction Energie Potentielle du système chimique en cours de dissociation, avec pour variables les coordonnées de réaction le long de la liaison qui se casse, les points stationnaires (géométrie à l'équilibre, état de transition) afin de connaître l'Energie d'activation de dissociation $E_{a}^{d}$ de la réaction. Un mécanisme réactionnel est d'autant plus probable, parmi ceux possibles, que l'Energie $E_{a}^{d}$ est faible.

La détermination de la fonction Energie Potentielle est réalisée par les méthodes de calcul de la Chimie Quantique. Compte tenu du nombre d'atomes des molécules étudiées et du caractère relatif des résultats que nous recherchons, nous utilisons les méthodes semiempiriques (AM1, PM3 dans les codes MOPAC6, AMPAC).

D'autre part, nous considérons que même si sous l'effet d'une onde de choc, une certaine population de molécules explosives se trouve dans un état électronique excité [2], la population majoritaire de molécules reste à l'état fondamental. Donc, nous traitons la décomposition des molécules en nous intéressant au paramètre moléculaire, l'Energie d'activation de dissociation, calculé dans l'état électronique fondamental.

Les hypothèses de calcul sont déterminées par une étude préliminaire sur une molécule de la famille des Nitramines, de plus petite taille que l'Hexogène, la DiméhylNitramine DMN $\left[\left(\mathrm{CH}_{3}\right)_{2} \mathrm{~N}-\mathrm{NO}_{2}\right]$. Ces hypothèses sont les suivantes :

- hamiltonien et paramétrisation AM1,

- description de la structure électronique du système chimique par des méthodes en couches ouvertes (UHF) pour la plupart des réactions, et par des méthodes en couches fermées (RHF) pour les réarrangements (élimination de HONO). Nous représentons sur la figure $\mathrm{n}^{\circ} 2$ le graphe obtenu pour la cassure $\mathrm{N}-\mathrm{NO}_{2}$ de la DiméthylNitramine.

L'utilisation de l'Interaction de Configurations (IC) permet de traiter correctement un tel problème de réactivité chimique par l'obtention d'une fonction d'onde très flexible et donc apte à représenter celle du système chimique tout au long de son évolution. Cependant nous estimons sa mise en oeuvre trop délicate pour convenir à notre étude à caractère systématique et pour laquelle nous recherchons des résultats relatifs,

optimisation de la position des autres atomes de la géométrie à chaque pas de la coordonnée de réaction 


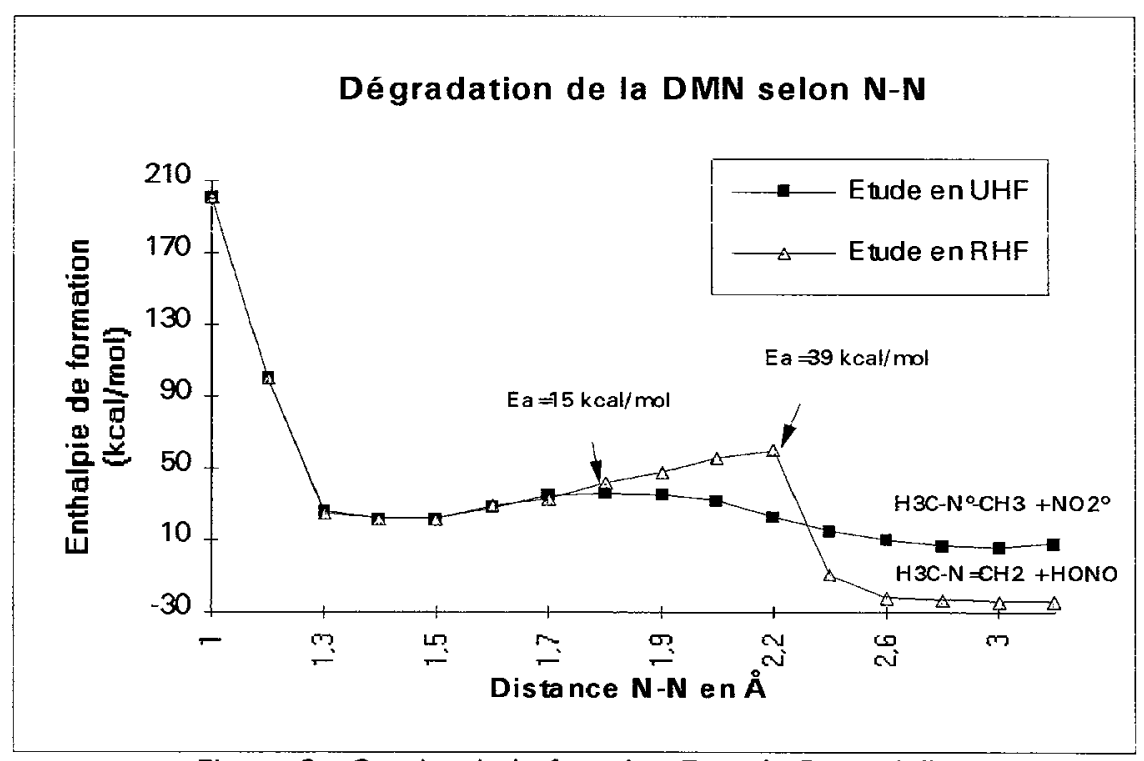

Figure 2 : Graphe de la fonction Energie Potentielle correspondant à la cassure $\mathrm{N}-\mathrm{NO}_{2}$ de la DMN

2.1.2 - Application à l'étude des mécanismes de décomposition de l'Hexogène (RDX)

Nous représentons sur la figure $\mathrm{n}^{\circ} 3$ le graphe de la fonction Energie Potentielle correspondant à la cassure de liaison simple $\mathrm{N}-\mathrm{NO}_{2}$ de l'Hexogène

$$
E_{p}(\text { kcal/mole) }
$$

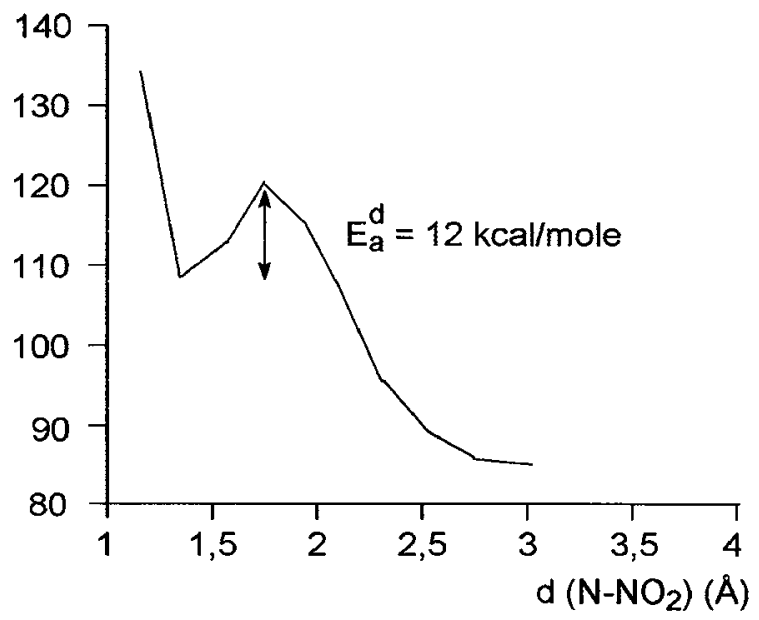

Figure 3 : Graphe de la fonction Energie Potentielle correspondant à la cassure $\mathrm{N}-\mathrm{NO}_{2}$ de l'Hexogène 
Nous indiquons sur la figure $n^{\circ} 4$ suivante la valeur des Energies d'activation de dissociation pour les réactions chimiques impliquées dans le premières étapes de la décomposition de RDX et dans la dissociation de la Méthylène-Nitramine $\left(\mathrm{H}_{2} \mathrm{C}=\mathrm{N}-\mathrm{NO}_{2}\right)$.

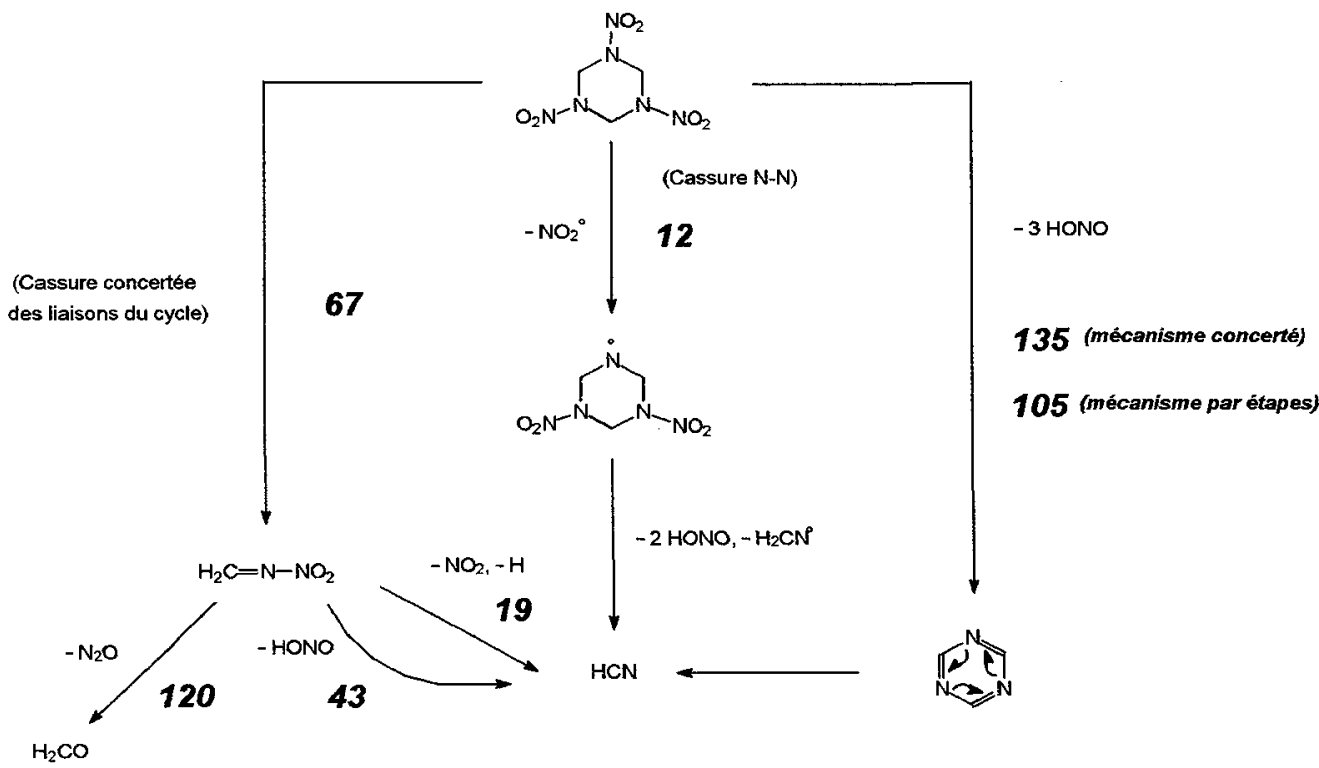

Figure 4 : Schéma de décomposition du RDX

Energies d'activation de dissociation ( $\mathrm{E}_{\mathrm{a}}^{\mathrm{d}} \mathrm{kcal} /$ mole $)$

Selon ces résultats, la voie préférentielle de dissociation du RDX est la cassure de liaison simple $\mathrm{N}_{-} \mathrm{NO}_{2}$, conduisant ensuite à $\mathrm{HCN}$. D'autre part, la décomposition de la méthylène-nitramine, espèce intermédiaire résultant de la cassure concertée des 3 liaisons $\mathrm{C}$ $\mathrm{N}$ du cycle, conduit également préférentiellement à $\mathrm{HCN}$ plutôt qu'à $\mathrm{H}_{2} \mathrm{CO}$. Ainsi, $\mathrm{NO}_{2}$ et HCN sont des espèces de petite taille qui se forment très tôt dans le processus. La formation de la triazine, par élimination de HONO après réarrangement intramoléculaire, semble peu probable. Par ordre d'importance décroissante, nous avons donc les espèces suivantes dans le milieu : $\left(\mathrm{NO}_{2}, \mathrm{HCN}\right),\left(\mathrm{N}_{2} \mathrm{O}, \mathrm{H}_{2} \mathrm{CO}\right)$, ( $\mathrm{HONO}$, triazine). Ces conclusions sont en accord global avec les éléments recueillis par notre recherche bibliographique.

\subsection{3 - Conclusion}

Nous établissons notre démarche théorique, afin d'approfondir notre connaissance des mécanismes réactionnels qui accompagnent la décomposition d'une molécule d'explosif à structure chimique donnée, par l'étude du RDX. Nous appliquons actuellement cette démarche à l'étude de la décomposition d'explosifs avec des formules chimiques et des comportements énergétiques différents : TATB, ANT, ONTA, DANTNP, pour déterminer les réactions les plus probables et les espèces formées prépondérantes en fonction de la structure moléculaire initiale. 
2.2 - Modélisation des spectres Infrarouge des espèces formées pendant la décomposition du RDX

La validation des résultats de l'étude théorique concernant les mécanismes réactionnels nécessite la mise en évidence des espèces chimiques formées dans le milieu en détonation. Pour cela, nous recherchons la détection des molécules par leur spectre IR (spectrométrie TRISP). L'interprétation des spectres expérimentaux est facilitée par leur modélisation qui présente deux aspects :

(fréquence et intensité),

- le calcul numérique des caractéristiques des modes de vibration

- la prise en compte des effets de forte pression et de température élevée du milieu en détonation sur les modes calculés.

\subsection{1 - Simulation numérique des spectres IR}

Nous nous intéressons aux spectres Infrarouges des espèces formées pendant la décomposition du RDX. Des spectres de référence existent pour les molécules HCN, $\mathrm{H}_{2} \mathrm{CO}, \mathrm{NO}_{2}, \mathrm{NO}, \ldots$ Par contre, les temps de vie des espèces intermédiaires sont trop courts pour qu'elles puissent être isolées et caractérisées par spectroscopie.

Pour la simulation numérique de ces spectres, nous utilisons les méthodes ab-initio de la Chimie Quantique au niveau Hartree-Fock et post-Hartree-Fock (MP2) dans le cadre des approximations classiques (Born - Oppenheimer et harmonicité).

Nous distinguons les systèmes à couches fermées $\left(\mathrm{H}_{2} \mathrm{CO}, \mathrm{HCN}, \mathrm{CO}\right)$ et ceux à couches ouvertes ou polarisables $\left(\mathrm{NO}_{2}, \mathrm{NO}\right)$. Pour ces derniers, l'existence d'un électron non apparié nécessite une flexibilité particulière de la fonction d'onde.

Nous présentons dans le tableau $\mathrm{n}^{\circ} 1$ ci-dessous les résultats concernant les fréquences et les intensités du spectre Infrarouge de la 1,3,5 Triazine, molécule représentative des systèmes à couches fermées. Ces résultats permettent de mettre en évidence les effets de base et de prise en compte de la corrélation électronique. Il apparaît qu'une hypothèse de calcul MP2/6-31G* conduit à des résultats satisfaisants.

\begin{tabular}{|c|c|c|c|c|c|c|c|c|c|}
\hline \multirow{2}{*}{\multicolumn{3}{|c|}{$\frac{4-31 G \text { basis set }}{\text { HF }}$}} & \multicolumn{6}{|c|}{$6-31 G^{*}$ basis set } & \multirow{3}{*}{ expérience } \\
\hline & & & & $\overline{\mathrm{HF}}$ & & & MP2 & & \\
\hline & Intensity & $\Delta(\%)$ & & Intensity & $\Delta(\%)$ & WFES & Intensity & $\Delta(\%)$ & \\
\hline 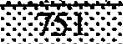 & 42 & 9,3 & & 46 & 10 & $6 \%$ & 38 & 0,1 & \\
\hline $80 \%$ & 52 & 8,3 & 836 & 64 & 13 & Aि & 32 & $-3,9$ & 739 \\
\hline Wato & 5 & 4,1 & $1+2$ & 2 & 5 & 60203 & 1 & 10 & 403 \\
\hline$\theta 30 \%$ & 2 & 12 & 1272 & 0,2 & 9 & $\theta+1$ & 0,04 & 3,8 & 167 \\
\hline 1519 & 213 & 12 & 1566 & 231 & 10 & 1469 & 112 & 4,2 & 1409 \\
\hline 480 & 474 & 16 & 16\% & 274 & 11,2 & 16396 & 154 & 3,8 & 1560 \\
\hline 340 & 74 & 11,4 & 344 & 30 & 12,8 & 3243 & 42 & 6,2 & 031 \\
\hline
\end{tabular}

Tableau $n^{\circ} 1$ : Comparaison des spectres IR expérimental et calculé de la Triazine 
Pour les systèmes à couches ouvertes, représentatifs des espèces radicalaires intermédiaires, les méthodes ab-initio testées ne fournissent pas des ordres de grandeur concluants. Cela est illustré par les résultats présentés dans le tableau $n^{\circ} 2$ pour $\mathrm{NO}_{2}$. Quelle que soit l'hypothèse retenue, un écart important subsiste entre les fréquences calculées et expérimentales des trois modes.

\begin{tabular}{|c|c|c|c|}
\hline & 01 & u2 & $v 3$ \\
\hline $3-21 G$ & $1280(-341)$ & $1253(-105)$ & $748(-9)$ \\
\hline $4-31 G$ & $1570(+51)$ & $1442(+84)$ & $781(+24)$ \\
\hline $6-31 \mathrm{G}^{*}$ & $1880(+259)$ & $1612(+254)$ & $832(+50)$ \\
\hline $6-31+G^{*}$ & $1865(+244)$ & $1605(+247)$ & $832(+50)$ \\
\hline $\mathrm{MP2/6-31G}$ * & $2272(+651)$ & $1381(+23)$ & $752(-5)$ \\
\hline $\mathrm{MP} 2 / 6-31+\mathrm{G}^{*}$ & $2309(+688)$ & $1370(+12)$ & $752(-5)$ \\
\hline $\begin{array}{l}\text { CISD/6- } \\
31+\mathrm{G}^{*}\end{array}$ & $2505(+884)$ & $1492(+134)$ & $782(+25)$ \\
\hline exp. & 1621 & 1358 & 757 \\
\hline
\end{tabular}

Tableau $\mathrm{n}^{\circ} 2$ : Comparaison des spectres IR expérimental et calculé de $\mathrm{NO}_{2}\left(u\right.$ en $\left.\mathrm{cm}^{-1}\right)$

Actuellement, afin de mieux prendre en compte les effets de corrélation dans le calcul de la structure électronique des espèces formées (systèmes à couches ouvertes ou polarisables), nous utilisons les techniques basées sur la Théorie de la Fonctionnelle Densité. Les premiers résultats obtenus avec NO sont encourageants.

2.2.2 - Prise en compte des effets de forte pression et température élevée sur les modes de vibration

La forte pression et la température élevée du milieu en détonation entraînent une modification des caractéristiques (Intensité, Fréquence) des modes de vibration calculés.

Afin de connaître l'évolution de ces modes, une étude est effectuée au LIMHPCNRS à VILLETANEUSE (93 - FRANCE) sur un banc haute pression pour suivre, jusqu'à $50 \mathrm{GPa}$ et $550^{\circ} \mathrm{C}$, l'évolution du spectre de vibration du $\beta$-HMX. Ce suivi systématique est réalisé par spectroscopie Raman. L'objectif est de collecter un ensemble de données expérimentales afin de développer une modélisation de ces effets.

Les résultats obtenus permettent de mettre en évidence, à température ambiante, trois transitions de phase se manifestant par l'apparition de nouveaux modes, des sauts de fréquence et des changements de pente dans l'évolution des modes en fonction de la pression. De plus, à température ambiante, il n'y a pas de modification chimique de la molécule jusqu'à $40 \mathrm{GPa}$. Les seules modifications dues à la pression sont d'ordre structural ou conformationnel. 


\section{3 - Approche expérimentale}

Pour la validation des résultats de l'étude théorique, l'intérêt de la spectrométrie TRISP, par rapport aux autres techniques expérimentales existantes, est de sonder directement le milieu en détonation.

Le schéma de l'expérience est représenté sur la figure $n^{\circ} 5$.

Le principe du fonctionnement de la chaîne d'analyse, décrit sur la figure $n^{\circ} 6$, est le suivant : un faisceau Infrarouge large bande pulsé est généré par effet SERS dans une cellule de conversion de fréquence remplie de métal alcalin $(\mathrm{Rb})$. Le faisceau IR traverse le milieu en détonation où il est modulé par l'absorption des espèces chimiques formées. Il est ensuite reconverti en faisceau visible par effet SERS et par mélange d'ondes dans une seconde cellule de conversion de fréquence. Il est analysé par un monochromateur associé à un détecteur à barrettes de photodiodes et à un analyseur multicanal. Le domaine spectral entre $2 \mu \mathrm{m}$ et $11 \mu \mathrm{m}$ est couvert par intervalles de quelques centaines de $\mathrm{cm}^{-1}$; le positionnement en longueur d'onde dépend du couple colorant/métal alcalin.

Actuellement, nous optimisons les paramètres expérimentaux afin de générer un faisceau IR d'énergie suffisante pour pouvoir sonder le milieu en détonation.

\section{Conclusion}

La compréhension des mécanismes réactionnels mis en jeu pendant la décomposition de l'explosif et la mise en évidence des espèces formées dans le milieu entraînent une meilleure connaissance de son comportement énergétique.

Par une approche chimique, et par le calcul des Energies d'activation de dissociation à l'aide des méthodes de calcul semi-empiriques de la Chimie Quantique, nous déterminons les principales réactions élémentaires qui se produisent pendant la décomposition. Nous recherchons une validation des résultats par la détection des produits dans le milieu en détonation par la Spectrométrie TRISP. La démarche a été d'abord appliquée au RDX.

Un autre aspect de la modélisation est le calcul théorique, par des techniques abinitio, des spectres IR des espèces formées afin de faciliter l'interprétation des spectres expérimentaux. La prise en compte des spectres IR des espèces intermédiaires nécessite l'utilisation de techniques basées sur la Théorie de la Fonctionnelle Densité.

En ce qui concerne la prise en compte des effets de pression et température élevées du milieu en détonation sur ces spectres, des expérimentations sont réalisées sur des espèces qui seront présentes dans les produits de détonation à l'aide d'une cellule à enclume de diamant. La modélisation des effets de $\mathrm{P}$ et $\mathrm{T}$ pourra ainsi être recalée sur des données expérimentales et extrapolée aux espèces transitoires. 


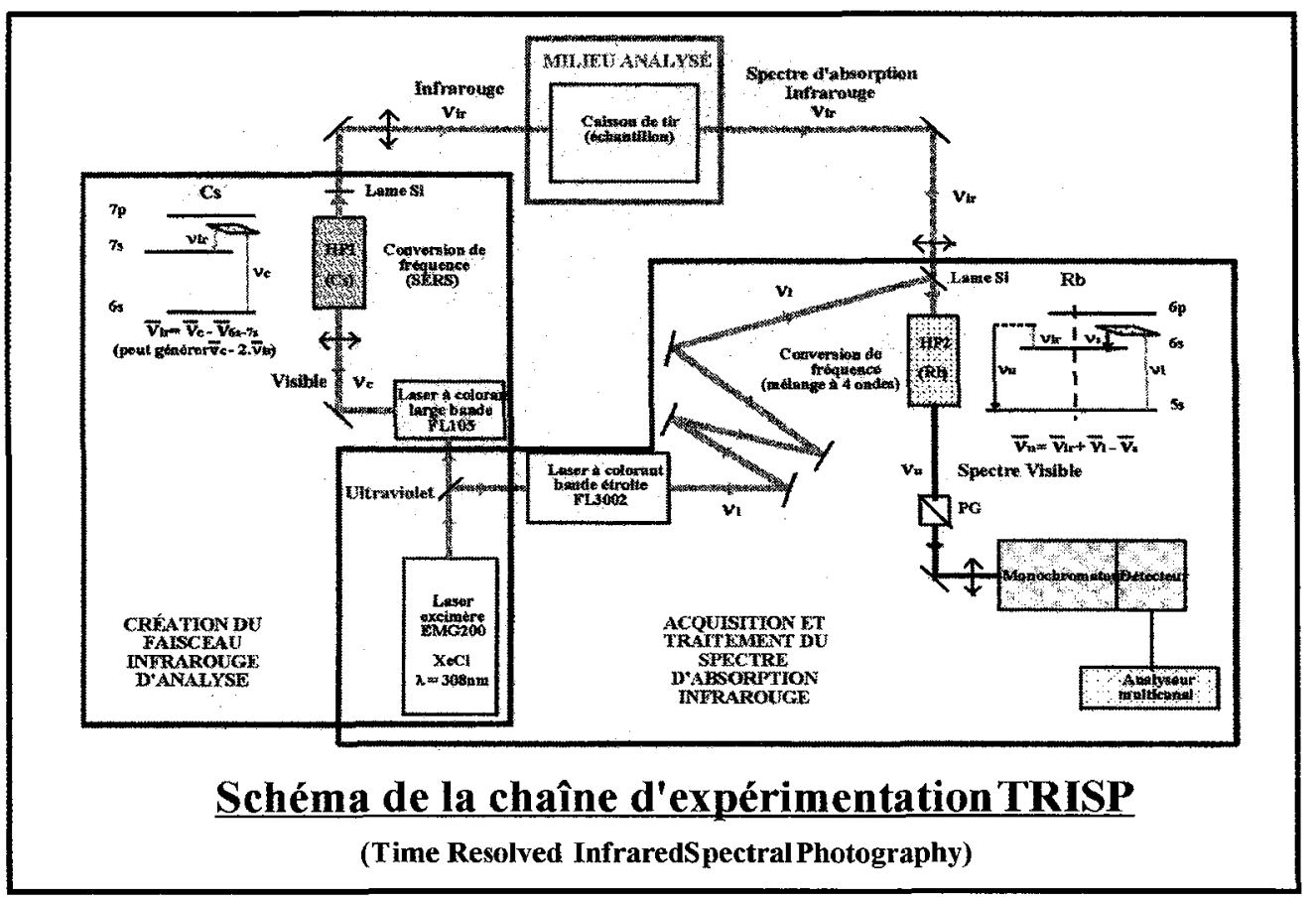

fig. $n^{\circ} 5$

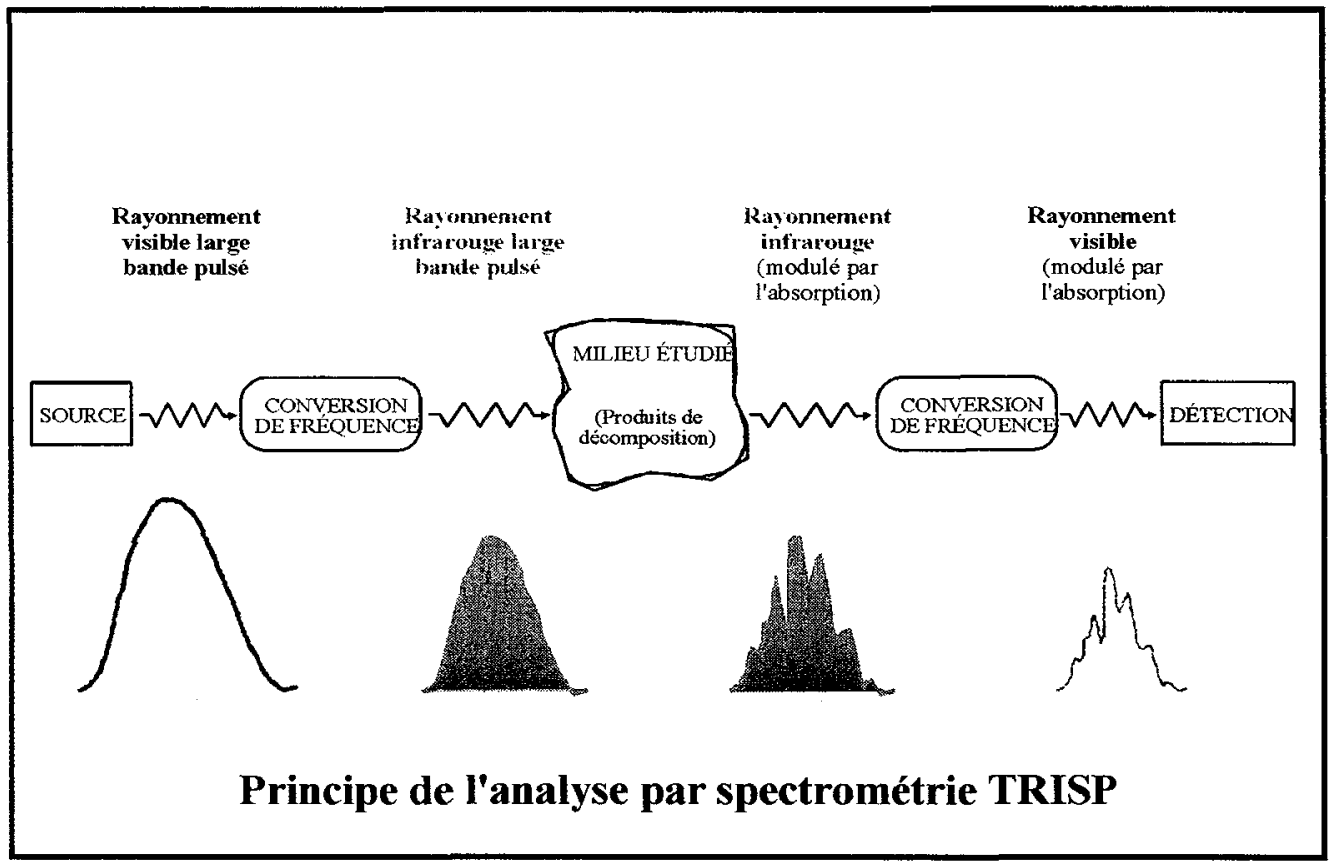

fig. $n^{\circ} 6$ 


\section{REFERENCES}

[1] A.M. RENLUND, SA SHEFFIELD, WM TROTT Time resolved Infra Red Spectral Photography : Study of shockinduced chemistry in $\mathrm{CS}_{2}$

Shock waves in condensed matter, p. 237, Spokane

Edited by Y.R. GUPTA, PLENUM PRESS (1985)

[2] A. DELPUECH - J. CHERVILLE - C. MICHAUD 7ème Symposium international sur la détonation

ANNAPOLIS (1981) 